\title{
MicroRNA-31 functions as an oncogenic microRNA in cutaneous squamous cell carcinoma cells by targeting RhoTBT1
}

\author{
NENGXING LIN, YU ZHOU, XIN LIAN and YATING TU \\ Department of Dermatology, Union Hospital, Tongji Medical College, Huazhong University of Science and Technology, \\ Wuhan, Hubei 430022, P.R. China
}

Received February 6, 2015; Accepted March 1, 2016

DOI: $10.3892 / 01.2017 .5554$

\begin{abstract}
Cutaneous squamous cell carcinoma (cSCC) is a malignancy of epidermal keratinocytes that is responsible for $20 \%$ of annual skin cancer-associated mortalities. Accumulating evidence demonstrates that the dysregulation of micro (mi)RNAs serves a significant role in the tumorigenesis and progression of human cSCC. MicroRNA-31 (miR-31) is upregulated in $\mathrm{cSCC}$ and is involved in cSCC development. However, the underlying mechanism remains unclear. The present study demonstrated that miR-31 is upregulated in the cSCC cell line, A-431, and that miR-31 expression contributes to the cell proliferation and invasion of cSCC. In addition, bioinformatics combined with dual luciferase reporter analysis was applied to determine that the tumor suppressor RhoTBT1 was a direct target of miR-31. In addition, miR-31 mimics reduced RhoBTB1 expression in A-431 cells. The results of MTT and Transwell assays demonstrated that knockdown of RhoBTB1 by short interfering RNA induced cell proliferation and invasion in A-431 cells. These results indicated that suppression of RhoBTB1 may be involved in cSCC tumorigenesis, which was directly affected by miR-31. In conclusion, the present study provides evidence that miR-31 acts as an oncogene through direct repression of RhoTBT1 expression in cSCC cancer, suggesting a potential application of miR-31 in prognosis prediction and its therapeutic application in $\mathrm{cSCC}$.
\end{abstract}

\section{Introduction}

Cutaneous squamous cell carcinoma (cSCC) is one of the most common types of skin cancer leading to $20 \%$ of annual skin cancer-associated mortalities $(1,2)$. Although the risk of local recurrence and metastasis of cSCC are well characterized, the

Correspondence to: Professor Nengxing Lin, Department of Dermatology, Union Hospital, Tongji Medical College, Huazhong University of Science and Technology, 1277 Jiefangdadao, Wuhan, Hubei 430022, P.R. China

E-mail: LinNengxing2014@163.com

Key words: cutaneous squamous cell carcinoma, miR-31, RhoTBT1, cell proliferation, invasion molecular pathogenesis of this particular tumor type remain unclear. As increasing numbers of mortalities occur due to $\mathrm{cSCC}$, it is urgent to clarify the molecular mechanisms of this type of cancer and to develop novel and more effective treatment strategies against this malignancy.

MicroRNA (miRNA), a class of naturally occurring, 17-25 nucleotide small noncoding RNA, regulates the expression of genes through binding to the $3^{\prime}$ untranslated regions (3'-UTR) of target mRNAs. MiRNAs have emerged as key factors involved in a number of biological processes, including development, differentiation, cell proliferation, and tumorigenesis (3-5). Previous studies have shown that alterations in miRNA genes lead to tumor formation, and miRNAs that regulate either tumor suppression or tumor formation have been identified (6-8). Previous studies have also identified a number of dysregulated miRNAs were observed in cSCC $(9,10)$. Zhou et al (11) demonstrated that miR-365 was overexpressed in both cells and clinical specimens of cSCC (11). The reduced expression of the miR-193b/365a cluster observed during tumor progression suggests a tumor suppressor role in cSCC (12). MiR-199a inhibits cSCC cell proliferation and migration by regulating CD44-Ezrin signaling (13).

Accumulating studies have shown that miR-31 expression is correlated with metastasis; however, the functional role of this miRNA is extremely complex as it may function as an oncogenic or a tumor-suppressive miRNA depending on the cellular contexts (14-16). Previous studies have reported that miR-31 is upregulated in cervical cancer $(15,17,18)$, and oesophageal squamous cell carcinoma (19), but downregulated in breast cancer $(20,21)$, bladder cancer (16), malignant mesothelioma (22), gastric cancer (23) and pancreatic cancer (24). Another study has demonstrated that miR-31 is overexpressed in $\mathrm{CSCC}$ and that it regulates cancer-associated phenotypes of cSCC (25), but the mechanisms behind its potential involvement on proliferation and tumor cell invasion remain unclear.

In the present study, the expression of miR-31 was investigated in $\mathrm{cSCC}$, and the downstream targets of miR-31 were also explored. The role of miR-31 in cSCC was also analyzed in relation to tumorigenesis and invasiveness.

\section{Materials and methods}

Cell culture and transfection. A cSCC cell line (A-431) and a normal skin cell line $(\mathrm{HaCaT})$ were obtained from the 
American type culture collection (ATCC, Manassas, VA, USA) and cultivated in RPMI-1640 medium with $10 \%$ fetal bovine serum (both Gibco; Thermo Fisher Scientific, Inc., Waltham, MA, USA). All cells were cultured in $95 \%$ air and $5 \% \mathrm{CO}_{2}$ at $37^{\circ} \mathrm{C}$.

A-431 cells were seeded and transfected at a density of $5 \times 10^{5}$ cells with miR-31 mimics or inhibitors (Qiagen Operon, Alameda, CA, USA), RhoBTB1 siRNA and control siRNA using Lipofectamine 2000 (Invitrogen; Thermo Fisher Scientific, Inc.) according to the manufacturer's instructions. A total of 24 or $48 \mathrm{~h}$ later, the cells were collected and subjected to further analysis.

$R N A$ extraction and reverse transcription-quantitative polymerase chain reaction ( $R T-q P C R)$. Total RNA was extracted from transfected A-431 cells using TRIzol reagent (Invitrogen, ThermoFisher Scientific, Inc.) and then reverse-transcribed into cDNA. RT-qPCR was performed using the SYBR Green qPCR Master Mix (Tiangen Biotech Co., Ltd., Beijing, China) on an ABI 7300 PCR machine (Applied Biosystems, Inc., Foster, CA, USA). The sequences of the primers used to detect miR-31 and U6 were as follows: miR-31, forward 5'-GGAGAG GCAAGATGCTGGCA-3'; U6, forward 5'-CGCAAGGAT GACACGCAAATTC-3'; and a universal downstream reverse primer, 5'-GTGCAGGGTCCGAGGT-3'. The primers used for detection of RhoBTB1 were as follows: forward 5'-GGA GTGAAGGAGCCTGTGAG-3'; and reverse 5'-TGCCAA TGAACCCCTTACTC-3'. qPCR cycling conditions were as follows: $95^{\circ} \mathrm{C}$ for $10 \mathrm{~min}$, and then $95^{\circ} \mathrm{C}$ for $15 \mathrm{sec}$ and $50^{\circ} \mathrm{C}$ for $2 \mathrm{~min}$, for 40 cycles, followed by $60^{\circ} \mathrm{C}$ for $1 \mathrm{~min}$. The melting curve was $65-95^{\circ} \mathrm{C}$. The relative mRNA expression levels were calculated as $2^{-\Delta \Delta C q}$ and were normalized against U6.

Luciferase reporter assays. A-431 cells were seeded into a 24 -well plate at a density of $2.5-3 \times 10^{4}$ cells/well), after $24 \mathrm{~h}$ the cells were co-transfected with Renilla luciferase and luciferase reporter plasmids containing miR-31 or vector control and the wild-type or mutated target gene 3'-UTR using Lipofectamine 2000 (Invitrogen, ThermoFisher Scientific, Inc.). A total of $48 \mathrm{~h}$ after transfection, the luciferase activities were measured using a dual-luciferase reporter assay system (Promega Corporation, Madison, WI, USA). Firefly luciferase activities were normalized to Renilla luciferase activity.

Western blotting. The cells were washed with phosphate-buffered saline (PBS), and lysed with ice-cold RIPA (Sigma-Aldrich, St. Louis, MO, USA). Total protein $(60 \mu \mathrm{g})$ was extracted from transfected A-431 cells and separated on 10\% SDS-polyacrylamide gels for RhoBTB1 and $\beta$-actin detection. Anti-RhoBTB1 (catalog no. AV41883; $1: 1,000$ dilution) and anti- $\beta$-actin (catalog no. SAB2100037; 1:1,000 dilution) antibodies were purchased from Sigma-Aldrich. $\beta$-actin was used as loading control. The protein in the gels was transferred to nitrocellulose membranes, blocking was performed using 5\% milk, and then the membranes were incubated with the indicated antibodies in recommended dilution overnight at $4^{\circ} \mathrm{C}$. Then the membranes were washed with $0.1 \mathrm{M}$ PBST and incubated with HRP-conjugated secondary antibody (goat anti-rabbit IgG, (H+L) HRP conjugate; catalog no. A0545; Sigma-Aldrich). The signals were visualized using ECL Substrates (GE Healthcare

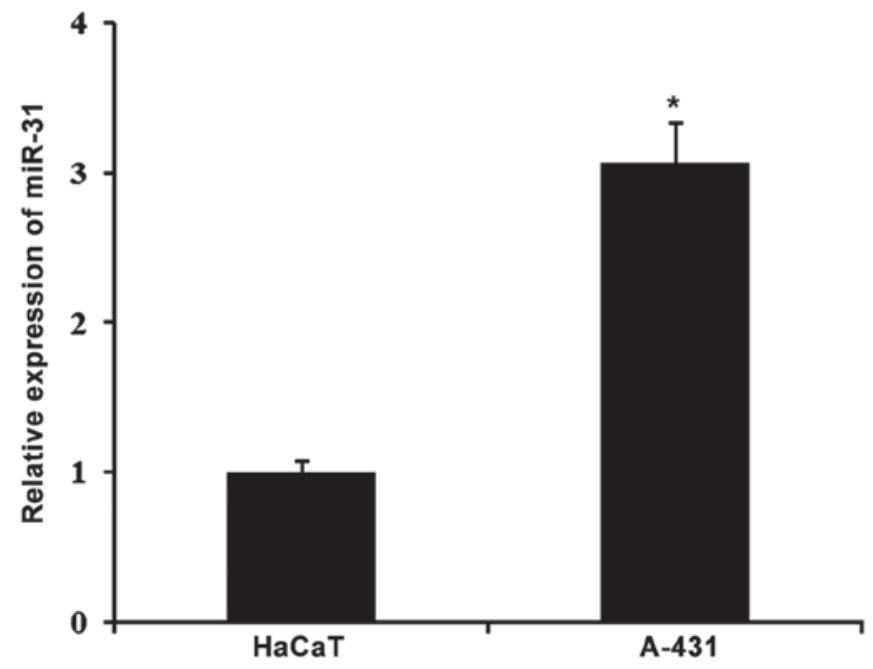

Figure 1. Quantification of the expression of miR-31 by reverse transcription-quantitative polymerase chain reaction assays in A-431 cells vs. HaCaT cells. miR-31 expression is upregulated in cutaneous squamous cell carcinoma cell lines. Error bars indicate standard deviation. ${ }^{*} \mathrm{P}<0.01$. miR-31, microRNA-31.

Life Sciences, Chalfont, UK), and quantified using Optiquant software (Packard Instrument Corporation, Meriden, CT, USA).

Cell viability assay. A cell viability assay was performed to investigate the effect of miR-31 or RhoBTB1 expression on the proliferation of A-431 cells. Following transfection as above, 6,000 cells of each treatment group were plated in 96-well plates in triplicate, and cell proliferation was assayed every $24 \mathrm{~h}$ using MTT (Beyotime Institute of Biotechnology, Haimen, China) according to the manufacturer's instructions.

Invasion assay. A- 431 cells were cultivated to $80 \%$ confluence in 12-well plates. Then, we observed the procedures of cellular growth at $24 \mathrm{~h}$. Cells were seeded in the Transwell migration chamber (Corning Inc., Corning, NY, USA) at a density of $2 \times 10^{6}$ cells and used to evaluate cell invasion. Then the cells that invaded across the Matrigel-coated membrane were counted under a light microscope (Olympus, Tokyo, Japan). All the experiments were repeated in triplicate.

Statistical analysis. Data are expressed as the mean \pm standard deviation and analyzed by Student's t-test. Statistical analysis was performed using SPSS 18.0 software (SPSS, Inc., Chicago, IL, USA). $\mathrm{P}<0.05$ was considered to indicate a statistically significant difference.

\section{Results}

miR-31 expression is upregulated in cSCC. A previous study revealed that miR-31 was dysregulated in cSCC tissues (25), therefore the present study examined miR-31 expression level in the cSCC cell line A-431 by using RT-qPCR. As shown in Fig. 1, RT-qPCR results demonstrated that compared with the HaCaT cell, miR-31 was significantly increased in A-431 cells $(\mathrm{P}<0.01)$, which was in accordance with the previous study (25). These results indicate that miR-31 may be involved in cSCC tumor progression. 

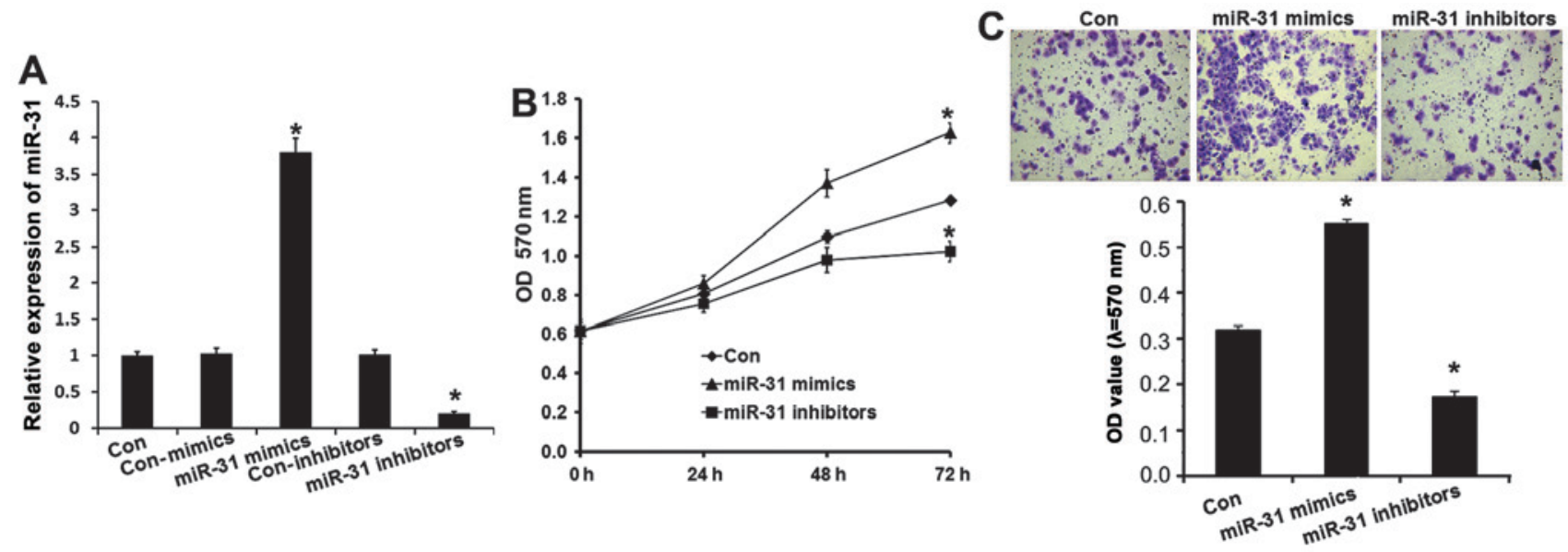

Figure 2. miR-31 affects cutaneous squamous cell carcinoma cell viability and invasion. (A) Reverse transcription-quantitative polymerase chain reaction analysis of miR-31 expression in A-431 cells. A-431 cells were transfected with miR-31 mimics, con-mimics, miR-31 inhibitors or con-inhibitors as indicated. (B) Cell viability was detected at the indicated time-points post-transfection using MTT assays. (C) Cell invasion was detected by Transwell assay. Error bars indicate standard deviation. ${ }^{*} \mathrm{P}<0.01$ vs. control. miR-31, microRNA-31.

A mir-31 target sites in $3^{\prime}$ UTR of RhoBtB1

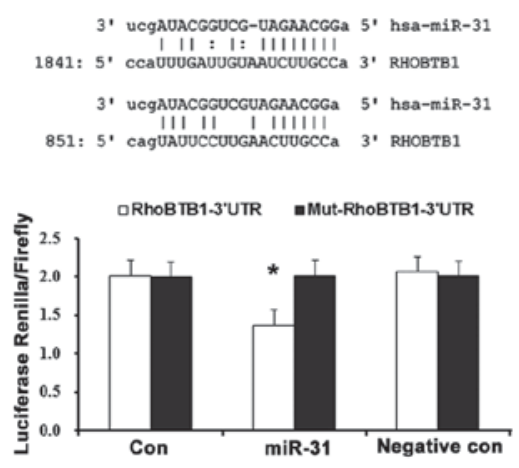

B

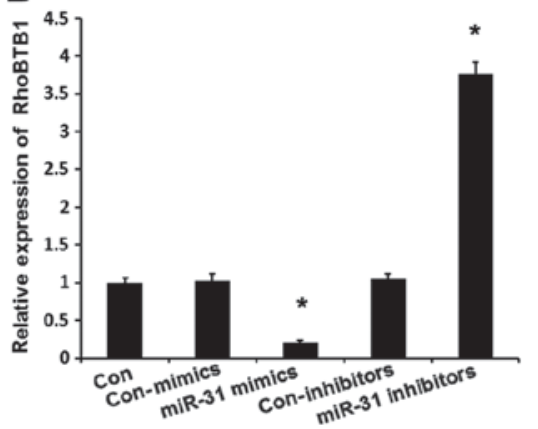

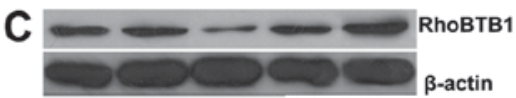

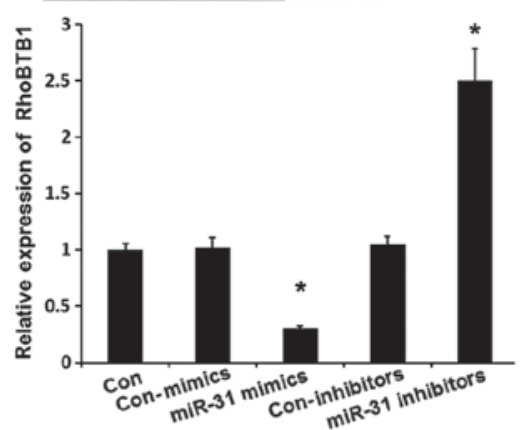

Figure 3. RhoBTB1 is a direct target of miR-31. (A) Sequence alignment of miR-31 and 3'-UTR of RhoBTB1. Luciferase reporter assay with co-transfection of wild-type or mutant RhoBTB1 and miR-31 mimics or miR-31 inhibitor or mimics-control or inhibitor-control or blank control in A-431 cells. (B) Reverse transcription-quantitative polymerase chain reaction analyses were performed to examine the effects of miR-31 on expression of RhoBTB1. (C) Western blotting was performed to determine RhoBTB1 protein levels in A-431 cells. Error bars represent \pm standard deviation and ${ }^{*} \mathrm{P}<0.01$ vs. control. miR-31, microRNA-31; 3'-UTR, 3'-untranslated region.
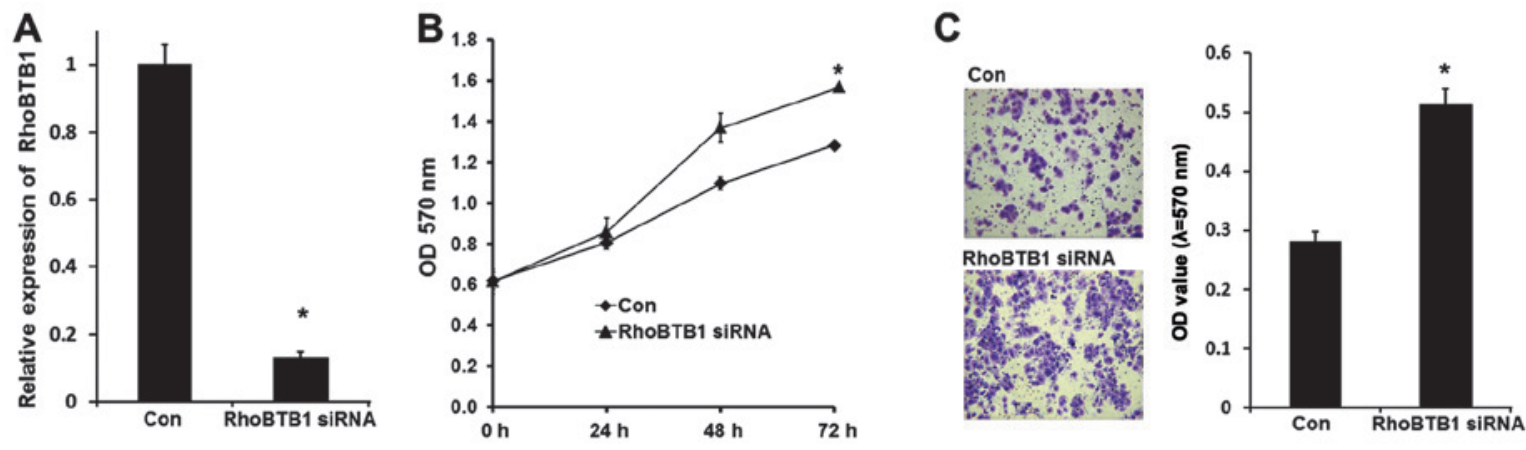

Figure 4. RhoBTB1 affects cutaneous squamous cell carcinoma cell viability and invasion. (A) Reverse transcription-quantitative polymerase chain reaction analysis of RhoBTB1 expression in A-431 cells. A-431 cells were transfected with RhoBTB1 siRNA as indicated. (B) Cell viability was detected at the indicated time-points post-transfection using MTT assays. (C) Cell invasion was detected by Transwell analysis. Error bars indicate standard deviation. " $\mathrm{P}<0.01$ vs. control. miR-31, microRNA-31; siRNA, small interfering.RNA.

miR-31 affects human cSCC cell viability and invasion. To further reveal the role of miR-31 in cSCC development, miR-31 mimics or inhibitors were transfected into A-431 cells to overexpress or silence miR-31 expression. As demonstrated in Fig. 2A, following transfection with miR-31 mimics, miR-31 expression was effectively upregulated $(\mathrm{P}<0.01)$, and miR-31 
expression was downregulated in A-431 cells after tranfection with miR31 inhibitors $(\mathrm{P}<0.01)$. An MTT assay demonstrated that overexpression of miR-31 significantly increased cell viability and inhibition of miR-31 reduced viability of A-431 cells (Fig. 2B), which indicated that miR-31 contributed to cSCC tumorigenesis.

To verify the involvement of miR-31 in cSCC tumorigenesis, a Transwell assay was performed to identify the effect of miR-31 on cSCC cell invasion. The results demonstrated that the invasion capabilities of A-431 cells was markedly increased in the miR-31 mimics group $(\mathrm{P}<0.01)$ and reduced in the miR-31 inhibitor group $(\mathrm{P}<0.01)$, indicating that miR-31 may induce A-431 cell invasion (Fig. 3C). In conclusion, the results demonstrated that miR-31 contributed to $\mathrm{cSCC}$ cell viability and invasion, which further indicated that miR-31 may be involved cSCC development.

RhoBTB1 is a direct target of $m i R-31$ in $c S C C$. In order to elucidate the underlying molecular mechanism of miR-31 action, a bioinformatic analysis was performed using mirco-RNA. org (http://www.microrna.org/microrna/home.do) to predict the possible target genes of miR-31. It was demonstrated that RhoBTB1 contained two theoretical miR-31 binding sites in its 3' UTR (Fig. 3A). To demonstrate whether RhoBTB1 was directly targeted by miR-31, a luciferase reporter gene assay was performed in A-431 cells. As presented in Fig. 3B, co-transfection of miR-31 suppressed the luciferase activity of the reporter containing the wild-type RhoBTB1 3'-UTR sequence, but failed to inhibit that of mutated RhoBTB1 by dual-luciferase reporter assay. These data indicated that miR-31 could directly target the 3'-UTR sequences of RhoBTB1. Additionally, in A-431 cells, the expression of RhoBTB1 was suppressed by miR-31 mimics transfection (Fig. 3B; P<0.01), while RhoBTB1 expression was enhanced by miR-133a inhibitor, which was also confirmed by western blot analysis (Fig. $3 \mathrm{C} ; \mathrm{P}<0.01$ ). These results demonstrated that endogenous RhoBTB1 expression is directly targeted and regulated by miR-31 and suggested that miR-31 upregulation in cSCC may reduce the expression of RhoBTB1.

Inhibition of RhoBTB1 is responsible for the tumor promoting effects of miR-31. To further confirm that miR-31-induced cSCC progression is mediated by RhoBTB1, RhoBTB1 expression was knocked down in A-431 cells by siRNA. As shown in Fig. 4A, RhoBTB1 mRNA was effectively inhibited after RhoBTB1 siRNA were transfected, and the MTT assay results demonstrated that A-431 cell proliferation was induced with suppression of RhoBTB1. Transwell invasion assay results demonstrated that inhibition of RhoBTB1 promoted A-431 cell invasion (Fig. 4B). These data indicated that miR-31 promoted tumor development at least partly through suppressing tumor supressor RhoBTB1.

\section{Discussion}

Over the last decade, accumulating evidence has demonstrated that miRNAs are involved in the pathogenesis of a number of human diseases, including cancer. miR-31 may act as an oncogenic or a tumour-suppressive miRNA and serves important roles in tumorigenesis and the progression of chemotherapy resistance $(14,22,26)$. For example, downregulation of miR-31 confers resistance to chemotherapy-induced apoptosis in prostate cancer cells (26), and it has been reported that miR-31 acts as an oncogenic miRNA (oncomir) in lung cancer by targeting specific tumor suppressors LATS2 and PPP2R2A (14). MiRNA-31 functions as an oncogenic MicroRNA in human colorectal cancer by repressing RAS p21 GTPase activating protein 1 (RASA1), SATB2 and HIF-1 $\alpha$ (FIH-1) $(15,18,27)$. In the present study, it was demonstrated that miR-31 was significantly upregulated in $\mathrm{cSCC}$. This result was consistent with the findings of previous studies that miR-31 is overexpression in cSCC and induced cancer-associated phenotypes of cSCC (25). To examine the effect of miR-31 on cSCC proliferation and invasion, miR-31 mimics and inhibitors were transfected into A-431 cells to overexpress and knockdown miR-31. MTT assay results showed that A-431 cell proliferation was increased after miR-31 mimics transfection and decreased after miR-31 inhibitor transfection (Fig. 2A). The ability of cell invasion was greatly increased by miR-31 mimics and decreased by miR-31 inhibitor (Fig. 2B). These results suggest that miR-31 acts primarily as an oncogene in cSCC.

RhoTBT1 belongs to RhoBTB subfamily which are atypical members of the Rho family of small GTPases. The RhoBTB subfamily is composed of three members, RhoTBT1, RhoTBT2 and RhoTBT3 (28). RhoTBT2 may act as a tumor suppressor; it has been reported that lack of RHOBTB2 transcripts results in growth inhibition in breast cancer (28). Previous studies have also found high rates of loss of heterozygosity at the RHOTBT2 locus in gastric tumors and bladder tumors $(29,30)$. Similarly to RhoTBT2, RhoTBT1 was also recently reported to be a tumor supressor in a study on head and neck cancer and colon cancer $(31,32)$. However, analysis of RhoTBT2 in cSCC has not yet been reported. In the present study, RhoTBT2 was also identified to be a direct target of miR-31 in cSCC and miR-31 upregulation in cSCC might suppress RhoBTB1 expression. To further examine whether the depressed RhoBTB1 was responsible for the tumor promoting effects of miR-31, RhoBTB1 was silenced by siRNA, as indicated in Fig. 4, suppression of RhoTBT1 in A-431 induced cell proliferation, which was consistent with the function of miR-31 mimics. The knockdown of RhoTBT1 also promoted A-431 cells invasion.

In conclusion, the present study suggests high levels of miR-31 are involved in cSCC tumorigenesis, and tumor supressor RhoBTB1 was identified as a direct target of miR-31. Overexpression of miR-31 promotes tumor proliferation through reducing the expression of RhoBTB1. These observations shed new light on mechanisms underlying development of cSCC and supply potential novel therapeutic targets in inhibiting $\mathrm{CSCC}$ tumorigenesis.

\section{References}

1. Alam M and Ratner D: Cutaneous squamous-cell carcinoma. N Engl J Med 344: 975-983, 2001.

2. Ratushny V, Gober MD, Hick R, Ridky TW and Seykora JT: From keratinocyte to cancer: The pathogenesis and modeling of cutaneous squamous cell carcinoma. J Clin Invest 122: 464-472, 2012.

3. Bushati N and Cohen SM: MicroRNA functions. Annu Rev Cell Dev Biol 23: 175-205, 2007.

4. Zhang Y, Yang Q and Wang S: MicroRNAs: A new key in lung cancer. Cancer Chemother Pharmacol 74: 1105-1111, 2014. 
5. Xue J, Niu J, Wu J and Wu ZH: MicroRNAs in cancer therapeutic response: Friend and foe. World J Clin Oncol 5: 730-743, 2014.

6. Kent OA and Mendell JT: A small piece in the cancer puzzle: MicroRNAs as tumor suppressors and oncogenes. Oncogene 25: 6188-6196, 2006.

7. Acunzo M, Romano G, Wernicke D and Croce CM: MicroRNA and cancer-a brief overview. Adv Biol Regul 57: 1-9, 2015.

8. Lee JW, Choi CH, Choi JJ, Park YA, Kim SJ, Hwang SY, Kim WY, Kim TJ, Lee JH, Kim BG and Bae DS: Altered MicroRNA expression in cervical carcinomas. Clin Cancer Res 14: 2535-2542, 2008.

9. Bruegger C, Kempf W, Spoerri I, Arnold AW, Itin PH and Burger B: MicroRNA expression differs in cutaneous squamous cell carcinomas and healthy skin of immunocompetent individuals. Exp Dermatol 22: 426-428, 2013.

10. Sand M, Skrygan M, Georgas D, Sand D, Hahn SA, Gambichler T, Altmeyer P and Bechara FG: Microarray analysis of microRNA expression in cutaneous squamous cell carcinoma. J Dermatol Sci 68: 119-126, 2012

11. Zhou M, Liu W, Ma S, Cao H, Peng X, Guo L, Zhou X, Zheng L, Guo L, Wan M, et al: A novel onco-miR-365 induces cutaneous squamous cell carcinoma. Carcinogenesis 34: 1653-1659, 2013.

12. Gastaldi C, Bertero T, Xu N, Bourget-Ponzio I, Lebrigand K, Fourre S, Popa A, Cardot-Leccia N, Meneguzzi G, Sonkoly E, et al: MiR-193b/365a cluster controls progression of epidermal squamous cell carcinoma. Carcinogenesis 35: 1110-1120, 2014.

13. Wang SH, Zhou JD, He QY, Yin ZQ, Cao K and Luo CQ: MiR-199a inhibits the ability of proliferation and migration by regulating CD44-Ezrin signaling in cutaneous squamous cell carcinoma cells. Int J Clin Exp Pathol 7: 7131-7141, 2014.

14. Liu X, Sempere LF, Ouyang H, Memoli VA, Andrew AS, Luo Y, Demidenko E, Korc M, Shi W, Preis M, et al: MicroRNA-31 functions as an oncogenic microRNA in mouse and human lung cancer cells by repressing specific tumor suppressors. J Clin Invest 120: 1298-1309, 2010

15. Sun D, Yu F, Ma Y, Zhao R, Chen X, Zhu J, Zhang CY, Chen J and Zhang J: MicroRNA-31 activates the RAS pathway and functions as an oncogenic MicroRNA in human colorectal cancer by repressing RAS p21 GTPase activating protein 1 (RASA1). J Biol Chem 288: 9508-9518, 2013.

16. Wang S, Li Q, Wang K, Dai Y, Yang J, Xue S, Han F, Zhang Q, Liu J and Wu W: Decreased expression of microRNA-31 associates with aggressive tumor progression and poor prognosis in patients with bladder cancer. Clin Transl Oncol 15: 849-854, 2013

17. Xu XM, Qian JC, Deng ZL, Cai Z, Tang T, Wang P, Zhang KH and Cai JP: Expression of miR-21, miR-31, miR-96 and miR-135b is correlated with the clinical parameters of colorectal cancer. Oncol Lett 4: 339-345, 2012

18. Yang MH, Yu J, Chen N, Wang XY, Liu XY, Wang S and Ding YQ: Elevated microRNA-31 expression regulates colorectal cancer progression by repressing its target gene SATB2. PLoS One 8: e85353, 2013.

19. Zhang T, Wang Q, Zhao D, Cui Y, Cao B, Guo L and Lu SH: The oncogenetic role of microRNA-31 as a potential biomarker in oesophageal squamous cell carcinoma. Clin Sci (Lond) 121: 437-447, 2011
20. Viré E, Curtis C, Davalos V, Git A, Robson S, Villanueva A, Vidal A, Barbieri I, Aparicio S, Esteller M, et al: The breast cancer oncogene EMSY represses transcription of antimetastatic microRNA miR-31. Mol Cell 53: 806-818, 2014.

21. Körner C, Keklikoglou I, Bender C, Wörner A, Münstermann E and Wiemann S: MicroRNA-31 sensitizes human breast cells to apoptosis by direct targeting of protein kinase $\mathrm{C}$ epsilon (PKCepsilon). J Biol Chem 288: 8750-8761, 2013.

22. Ivanov SV, Goparaju CM, Lopez P, Zavadil J, Toren-Haritan G, Rosenwald S, Hoshen M, Chajut A, Cohen D and Pass HI: Pro-tumorigenic effects of miR-31 loss in mesothelioma. J Biol Chem 285: 22809-22817, 2010.

23. Guo J, Miao Y, Xiao B, Huan R, Jiang Z, Meng D and Wang Y: Differential expression of microRNA species in human gastric cancer versus non-tumorous tissues. J Gastroenterol Hepatol 24: 652-657, 2009.

24. Papaconstantinou IG, Manta A, Gazouli M, Lyberopoulou A, Lykoudis PM, Polymeneas G and Voros D: Expression of microRNAs in patients with pancreatic cancer and its prognostic significance. Pancreas 42: 67-71, 2013.

25. Wang A, Landén NX, Meisgen F, Lohcharoenkal W, Ståhle M, Sonkoly E and Pivarcsi A: MicroRNA-31 is overexpressed in cutaneous squamous cell carcinoma and regulates cell motility and colony formation ability of tumor cells. PLoS One 9: e103206, 2014

26. Bhatnagar N, Li X, Padi SK, Zhang Q, Tang MS and Guo B: Downregulation of miR-205 and miR-31 confers resistance to chemotherapy-induced apoptosis in prostate cancer cells. Cell Death Dis 1: e105, 2010

27. Chen T, Yao LQ, Shi Q, Ren Z, Ye LC, Xu JM, Zhou PH and Zhong YS: MicroRNA-31 contributes to colorectal cancer development by targeting factor inhibiting HIF-1 $\alpha$ (FIH-1). Cancer Biol Ther 15: 516-523, 2014.

28. Hamaguchi M, Meth JL, von Klitzing C, Wei W, Esposito D, Rodgers L, Walsh T, Welcsh P, King MC and Wigler MH: DBC2, a candidate for a tumor suppressor gene involved in breast cancer. Proc Natl Acad Sci USA 99: 13647-13652, 2002.

29. Knowles MA, Aveyard JS, Taylor CF, Harnden P and Bass S: Mutation analysis of the 8p candidate tumour suppressor genes DBC2 (RHOBTB2) and LZTS1 in bladder cancer. Cancer Lett 225: 121-130, 2005.

30. Cho YG, Choi BJ, Kim CJ, Song JH, Zhang C, Nam SW, Lee JY and Park WS: Genetic analysis of the DBC2 gene in gastric cancer. Acta Oncol 47: 366-371, 2008

31. Beder LB, Gunduz M, Ouchida M, Gunduz E, Sakai A, Fukushima K, Nagatsuka H, Ito S, Honjo N, Nishizaki K and Shimizu K: Identification of a candidate tumor suppressor gene RHOBTB1 located at a novel allelic loss region 10q21 in head and neck cancer. J Cancer Res Clin Oncol 132: 19-27, 2006.

32. Xu RS, Wu XD, Zhang SQ, Li CF, Yang L, Li DD, Zhang BG, Zhang Y, Jin JP and Zhang B: The tumor suppressor gene RhoBTB1 is a novel target of miR-31 in human colon cancer. Int J Oncol 42: 676-682, 2013. 